\title{
A review of geriatric care training in the undergraduate nursing and medical curricula at the University of KwaZulu-Natal, South Africa
}

\author{
K Naidoo, ${ }^{1} \mathrm{MB}$ ChB, MCFP (SA); F Waggie, ${ }^{2} \mathrm{PhD}$; J M van Wyk, ${ }^{3} \mathrm{PhD}$ \\ ${ }^{1}$ Discipline of Family Medicine, College of Health Sciences, University of KwaZulu-Natal, Durban, South Africa \\ ${ }^{2}$ Interprofessional Education Unit, University of the Western Cape, Cape Town, South Africa \\ ${ }^{3}$ Department of Clinical and Professional Practice, College of Health Sciences, University of KwaZulu-Natal, Durban, South Africa
}

Corresponding author: KNaidoo (naidook7@ukzn.ac.za)

\begin{abstract}
Background. The population in South Africa is ageing rapidly. However, health professionals are reportedly unprepared to provide quality care for the elderly population. A situational analysis of curricula is required to identify the extent to which current training addresses the needs of elderly populations. Objectives. To investigate the undergraduate medical and nursing curricula at a South African university regarding geriatric care training, and explore possible learning opportunities to enhance health professions education in geriatric care.

Methods. This descriptive exploratory study was conducted through document review and semi-structured interviews with health professions educators. Results. In both curricula, a problem-based learning approach was combined with classroom and bedside teaching. A wide range of geriatric topics was covered in each programme, four of which were common to both, i.e. falls, urinary incontinence, dementia and chronic non-communicable diseases. Nursing students were exposed to geriatric patients in multiple settings, while medical students saw geriatric patients mainly in hospitals and community clinics. Geriatric content in both programmes was integrated into other modules, and there was no independent assessment of geriatric competencies.

Conclusion. Although a multitude of geriatric learning objectives were included in both nursing and medical training programmes, there was limited coverage and a lack of discrete assessment in this field. Opportunities to enhance the current curricula include discrete assessment of geriatric care competencies, and increased interprofessional education. However, faculty development and additional resources would be required in both programmes.
\end{abstract}

Afr J Health Professions Educ 2020;12(3):130-133. https://doi.org/10.7196/AJHPE.2020.v12i3.1349

The lack of attention to geriatric care training in health professions curricula is concerning given the rapid increase in the number of people aged $\geq 60$ years in South Africa (SA).${ }^{[1]}$ Most older adults in SA access health services at primary care level. Nursing and medical graduates who are at the forefront of primary care services must be prepared to care for the increasing number of older adults needing care for chronic and age-related health conditions. However, studies indicate that the level of primary care provided to older adults in SA is inadequate, due to a lack of appropriate training of health professionals. ${ }^{[2,3]}$

Older adults are prone to multiple health conditions, and thus require co-ordinated care to preserve function and improve their quality of life..$^{[4]}$ Reports of polypharmacy and adverse drug reactions in elderly patients are ascribed to fragmented and inappropriate management of older adults. ${ }^{[5]}$ The World Health Organization highlights the need for greater interdisciplinary team skills among primary care providers to improve the care of older adults. ${ }^{[6]}$ While interprofessional collaboration and care are inherent in general nursing practice, there is little inclusion of interprofessional education (IPE) within nursing education to prepare graduates to provide co-ordinated care to older adults. ${ }^{[7]}$ It is therefore critical that geriatric care training be included in all undergraduate (UG) health professions curricula, and that training includes IPE. IPE occurs 'when two or more professions learn about, from and with each other to enable effective collaboration and improve health outcomes. ${ }^{[8]}$ Such a collaborative approach has the potential to reduce healthcare costs and care dependencies in the aged.
Despite the evident need for geriatric care training of nurses in SA, the SA Nursing Council has removed specialist gerontology from nursing curricula. Gerontology and geriatrics has also been neglected in undergraduate health professions education worldwide, and in sub-Saharan Africa in particular. ${ }^{[9]}$ Furthermore, nursing and medical undergraduate curricula in SA are developed and implemented independently of each other. It is therefore unclear whether these programmes adequately equip graduates to care for older adults as part of a multidisciplinary team in primary care.

A situational analysis was therefore required to identify the strengths, weaknesses, opportunities and threats of current nursing and medical curricula at the University of KwaZulu-Natal (UKZN), SA, in preparing graduates to care for older adults. This study aimed to analyse teaching and assessment of geriatric topics in the UG nursing and medical curricula at the UKZN, and explore potential opportunities to enhance IPE relevant to the care of older adults.

\section{Methods}

This mixed-methods study was conducted by a document review of module handbooks, study guides and an electronic curriculum platform used for the UG medical programme. In addition, semi-structured interviews were conducted with key informants $(n=5)$ involved in teaching and curriculum development. Data relating to each geriatric curriculum were analysed according to geriatric content, teaching methods and assessment per year 
of study (Tables 1 and 2). Content and thematic analyses were conducted. Ethical approval was obtained from the UKZN Biomedical Research Ethics Committee (ref. no. BE287/18) prior to data collection between July and September 2019. Both undergraduate nursing and medical degrees are offered in the College of Health Sciences at the UKZN. The programme for the nursing degree $(\mathrm{BN})$ is 4 years long, and the medical degree (MB ChB) 6 years.

\section{Results}

Health professions educators representing both curricula were found to be cognisant of the increasing number of older adults requiring primary healthcare services, and agreed on the importance of training students to care for older adults. However, they expressed concerns about increasing the teaching on geriatric care owing to time constraints of the programme and other priorities such as maternal and child health that have to be accommodated in the curriculum. Two main themes relevant to geriatric care training emerged from interviews with participants. These were patient-centredness and exposure of students to patients in authentic settings. Patient-centredness refers to understanding the patient as a whole person rather than merely a person with an illness. ${ }^{[10]}$ This was explicitly addressed in the nursing but not the medical curriculum:
'We have to teach them to be patient-centred. However, compassion fatigue is so common.' (Nurse educator, $>10$ years' teaching experience) 'They also do preventive and promotive health with the elderly, talking to them about diet, exercise, loneliness.' (Nurse educator, $>20$ years teaching experience)

Participants representing both programmes reflected that patient-centred care for older adults could be undermined by the negative influence of role models during clinical training:

'I think that they see from other sisters or doctors that the old are left in the corners to die.' (Nurse educator, $>15$ years' teaching experience)

An analysis of curriculum documents revealed that geriatric teaching in both disciplines involved a problem-based learning (PBL) approach (Tables 1 and 2). There is early exposure of students to paper-based cases, followed up with classroom and bedside teaching in later years of study. Both the nursing and medical curricula include teaching on a wide range of geriatric topics, of which four were common to both programmes, i.e. falls, dementia, urinary incontinence and chronic non-communicable diseases (Fig. 1).

Nursing students had clinical exposure to geriatric patients in multiple settings, such as in the community, primary care, residential facilities

Table 1. Geriatric teaching and learning in undergraduate nursing programme

\begin{tabular}{|c|c|c|c|}
\hline Year of study & Curriculum components & Details & Assessment \\
\hline \multirow[t]{2}{*}{1} & $\begin{array}{l}\text { Case-based discussions with PBL } \\
\text { approach }\end{array}$ & $\begin{array}{l}4 \times \text { patient studies covering falls, nutrition, wound care, urinary } \\
\text { incontinence, bereavement and palliative care, pain management, } \\
\text { activities of daily living }\end{array}$ & Written tests, short questions \\
\hline & Residential care nursing & $\begin{array}{l}1 \text { week allocation to residential care; history-taking and basic } \\
\text { nursing care }\end{array}$ & Written tests \\
\hline 2 & Community evaluation & No geriatric component & - \\
\hline 3 & Clinical geriatric experience & Allocation to wards, skills and procedures & Triple jump, OSCE, written test \\
\hline 4 & Psychogeriatrics & $\begin{array}{l}6 \text { weeks of mental health block spent with residential and } \\
\text { ambulatory geriatric patients with mental health conditions }\end{array}$ & $\begin{array}{l}\text { Reflective journals, } \\
\text { written cases, } \\
\text { triple jump, OSCE }\end{array}$ \\
\hline 4 & Primary healthcare & $\begin{array}{l}40 \text { hours in clinics - exposure to older patients with chronic } \\
\text { illnesses }\end{array}$ & $\begin{array}{l}\text { Reflective journals, } \\
\text { written cases, } \\
\text { triple jump, OSCE }\end{array}$ \\
\hline
\end{tabular}

Table 2. Geriatric teaching and learning in undergraduate medical programme

\begin{tabular}{|c|c|c|c|}
\hline Year of study & Curriculum components & Details & Assessment \\
\hline 1 & Principles of geriatrics & 1-hour lecture & MCQ \\
\hline 2 & None & None & \\
\hline 3 & $\begin{array}{l}\text { Lecture }+ \text { case-based discussions } \\
\text { with PBL approach }\end{array}$ & $\begin{array}{l}12 \text { lectures; case discussions covering prescribing for the elderly, legal } \\
\text { and ethical issues of ageing, physiological changes of ageing, dementia, } \\
\text { comprehensive geriatric assessment, urinary incontinence, falls, } \\
\text { infections, frailty, confusion, syncope and osteoporosis }\end{array}$ & MCQ, OSPE \\
\hline 4 & Lectures + ward rotations & $\begin{array}{l}\text { Lectures; clinical tutorials over } 12 \text { weeks covering dementia, } \\
\text { comprehensive geriatric assessment, falls, urinary incontinence, } \\
\text { infections, frailty, confusion, syncope and osteoporosis }\end{array}$ & Portfolio, DOSCE \\
\hline 5 & Ward rotations & $\begin{array}{l}6 \text { weeks of clinical experience with inpatients, } 1 \text { week of hospice } \\
\text { attachment; bedside teaching }\end{array}$ & MCQ, long case \\
\hline 6 & Ward rotations & 7 weeks of clinical experience with inpatients & $\begin{array}{l}\text { MCQ, DOSCE, portfolio, } \\
\text { long case }\end{array}$ \\
\hline
\end{tabular}




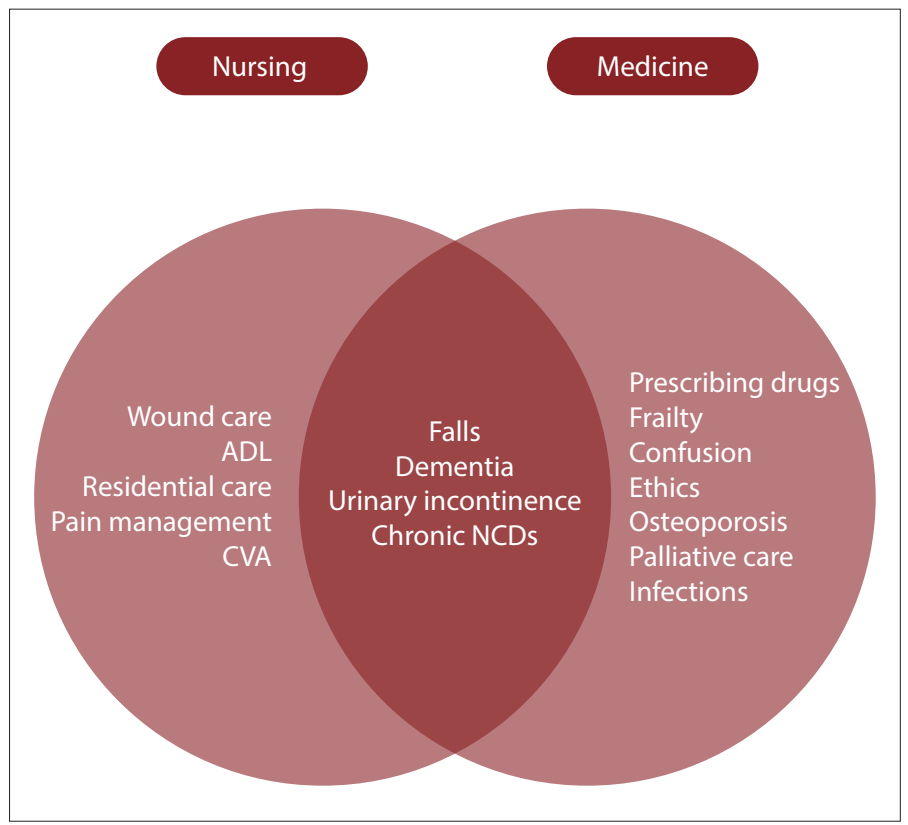

Fig. 1. Domains of geriatric care in the medical and nursing curricula. ( $A D L=$ activities of daily living; $C V A=$ cerebrovascular accident NCDs $=$ non-communicable diseases.)

and hospitals, whereas medical students saw geriatric patients mainly in hospitals and community clinics. The limited exposure of medical students to older adults in the community was acknowledged as a limitation of the medical curriculum:

'They need to see more ambulatory patients, with multiple conditions, and to be able to communicate with these patients.' (Medical educator, $>20$ years' teaching experience)

Teaching and assessment of geriatric content in both programmes was integrated into other modules, with no sub-minima applied in the assessment of geriatric topics. IPE was not included in either programme. Participants perceived that the large numbers of students enrolled in the already crowded curricula would hinder the implementation of IPE.

'Being problem-based, and with big classes, we can't address everything.' (Nurse educator, $>15$ years' teaching experience)

Health professions educators in both programmes said that they were not only inadequately prepared, but also inadequately resourced to implement IPE or to expand current geriatric teaching.

\section{Discussion}

This study highlighted the strengths and weaknesses of current geriatric training of nursing and medical students. Both curricula include teaching and learning on a range of geriatric topics, unlike many other training institutions in sub-Saharan Africa ${ }^{[11]}$ However, there were discrepancies between the geriatric topics covered in each curriculum. A notable finding of the study was the absence of collaboration with other health disciplines in the delivery of geriatric care training. This lack of collaboration and of a common foundation of geriatric conditions raises concerns not only about possible curricular gaps, but also about the ability of nursing and medical graduates to co-manage elderly patients in a patient-centred and efficient manner. There is an evident need for relevant stakeholders, including community representatives, to reach a consensus on the minimum core competencies in geriatric care for health professionals.

The PBL approach used in both curricula provides an effective means of educating students on core geriatric topics, and of addressing psychosocial and teamwork issues relevant to the care of older adults. Multiple common geriatric topics were identified in this study that involve management by a multidisciplinary team, such as dementia care. However, teaching on these topics did not include input from other disciplines. Models of IPE that include case-based PBL taught by educators from different disciplines such as nursing and medicine are feasible options to introduce IPE in this institution. ${ }^{[12]}$ However, health professions educators in both disciplines would need faculty development in order to effectively develop and implement IPE for geriatric care training.

Although there is some exposure to older adults in the community, the programme would benefit from greater exposure to community-dwelling older adults, as this has been shown to improve patient-centredness and attitudes of students towards older adults. ${ }^{[13]}$ Most older adults in SA are cared for at primary care level or in the community, and require professional health services that include preventive and promotive health services. Training should therefore occur in these authentic settings to prepare graduates to care for the majority of older adults in SA.

The lack of independent assessment of geriatric topics makes it difficult to ascertain whether graduates actually possess the necessary knowledge, skills and attitudes to care for older adults. Further investigation is required into graduate competencies in geriatric care.

Threats to geriatric care training in both programmes included time constraints and lack of confidence of educators to implement IPE. One suggestion to improve students' geriatric care competencies was to maximise the learning opportunities in facilities where interprofessional collaboration was practised. Unfortunately, this model of care was not practised at most of the clinical training sites. This study highlighted the need to expand current teaching and assessment relevant to the care of older adults in each discipline, and to ensure greater concordance between nursing and medical training programmes regarding primary care for older adults. This will enable graduates to work together in functional teams at primary care level to provide co-ordinated and quality care to older adults. Further investigation is required into the geriatric curricula of other health professions programmes in the college, such as occupational therapists and physiotherapists.

\section{Conclusion}

There are opportunities to maximise student learning and readiness to co-manage older patients in primary healthcare facilities by ensuring that students learn together in interprofessional teams. However, faculty development is required to upskill educators on IPE. Variable coverage of geriatric topics in each programme highlighted the need for consensus on the minimum geriatric core competencies for health professionals.

\section{Declaration. None.}

Acknowledgements. The authors would like to acknowledge the Sub-Saharan Africa-FAIMER Regional Institute (SAFRI) for their encouragement of this study. 
Author contributions. KN conceptualised and undertook the study under the supervision of JvW and FW. KN prepared the manuscript and JvW and FW reviewed and approved the final article.

Funding. None.

Conflicts of interest. None

1. Day C, Ndlovu N, Gray A. Health and related indicators 2018. S Afr Health Rev 2018(1):139-250. 2. Kalula SZ. The quality of health care for older persons in South Africa: Is there quality care? Conference paper. ESR Review: Econ Soc Rights S Afr 2011;12(1):22-25.

3. Abudu-Birresborn D, McCleary L, Puts M, Yakong V, Cranley L. Preparing nurses and nursing students to care for older adults in lower and middle-income countries: A scoping review. Int J Nursing Stud 2019;92:121-134. https://doi.org/10.1016/j.ijnurstu.2019.01.018

4. Aboderin IA, Beard JR. Older people’s health in sub-Saharan Africa. Lancet 2015:385(9968):e9-e11. https://doi. org/10.1016/S0140-6736(14)61602-0

5. Saka SA, Oosthuizen F, Nlooto M. Potential inappropriate prescribing and associated factors among older persons in Nigeria and South Africa. Int J Clin Pharm 2019;41(1):207-214. https://doi.org/10.21203/rs.2.15400/v1
6. World Health Organization. Interprofessional collaborative practice in primary health care: Nursing and midwifery perspectives. Geneva: WHO, 2013

7. Maree C, van Wyk H. Interprofessional health education to improve collaboration in the South African context: A realist view. Trends Nursing 2016;3(1):1-17. https://doi.org/10.14804/3-1-41

8. World Health Organization. Framework for action on interprofessional education and collaborative practice. Genev WHO, 2010. http://whqlibdoc.who.int/hq/2010/WHO_HRH_HPN_10.3_eng.pdf (accessed 17 March 2020).

9. Tanyi PL, Pelser A. The missing link: Finding space for gerontology content into university curricula in South Africa. Gerontol Geriatr Edu 2019;40(4):491-507. https://doi.org/10.1080/02701960.2018.1428579

10. Balint E. The possibilities of patient-centered medicine. J Royal Coll Gen Pract 1969;17(82):269.

11. Frost L, Liddie Navarro A, et al. Care of the elderly: Survey of teaching in an ageing sub-Saharan Africa. Gerontol Geriatr Educ 2015;36(1):14-29. https://doi.org/10.1080/02701960.2014.925886

12. Thompson S, Metcalfe K, Boncey K, et al. Interprofessional education in geriatric medicine: Towards best practice. A controlled before-after study of medical and nursing students. BMJ Open 2020;10(1):1-14. http://doi.org/10.1136 bmjopen-2017-018041

13. Mendoza De La Garza M, Tieu C, Schroeder D, Lowe K, Tung E. Evaluation of the impact of a senior mento program on medical students' geriatric knowledge and attitudes toward older adults. Gerontol Geriatr Educ 2018;39(3):316-325. https://doi.org/10.1080/02701960.2018.1484736

Accepted 19 June 2020. 